\title{
Purely Inseparable Extensions of Complete Intersections
}

\author{
By \\ Jeffrey LANG*
}

\section{$\S 0$. Introduction}

Let $R$ be a graded unique factorization domain and $h \in R$ a product of $q$ distinct homogeneous factors in $R$. Let $S=R[z] /\left(z^{m}-h\right)$. If $S$ is a Krull domain, then what is the divisor class group of $S$ ? In several cases the answer is that the divisor class group is a direct sum of $q-1$ copies of $Z / m Z$. For example, this is true if $R$ is a polynomial ring over a field and $h$ is a product of two variables ([7], page 58); also, if $R$ is a polynomial ring in two or more variables of characteristic $p \neq 0$, the factors of $h$ are homogeneous in $R$ and $m$ is a $p$-th power ([6], Proposition 3.11, page 627). In this paper the same phenomenon is verified in the following case.

Let $k$ be an algebraically closed field of characteristic $p \neq 0, Y \subset . A_{k}^{n}$ a complete intersection of dimension greater than one, and assume that the ideal that defines $Y$ is homogeneous. The above question is considered when $R$ is the coordinate ring of $Y$ and $h \in R$ is the image of a homogeneous element of $k^{[n]}$ of degree not divisible by $p$. We prove that if $m$ is a $p$-th power, then $C l(S)$ is a direct sum of $q-1$ copies of $Z / m Z$. This substantially improves a theorem obtained in a previous article ([5], page 569, Theorem 5.7).

\section{§1. Preliminaries}

This paper assumes familiarity with the subject of divisor class group of a Krull domain. Two excellent references are P. Samuel's 1964 Tata notes [7] and R. Fossum's, "The Divisor Class Group of a Krull Domain" [2]. If $A$ is a Krull domain, we denote the divisor class group of $A$ by $C l(A)$. If $Y=\operatorname{Spec} A$, we will refer to "the divisor class group of $Y$ ", denoted $C l(Y)$; by this we mean $C l(A)$. In this section we recall results from [2], [4] and [7].

Communicated by K. Saito, May 7, 1992.

1991 Mathematics Subject Classifications: 13B

* Dept. of Mathematics, KFUPM, Dhahran, Saudi Arabia.

On leave from the Mathematics Department, University of Kansas, Lawrence, Kansas, 66045 , U.S. A. 
Theorem 1. Let $S \subset T$ be Krull domains with $T$ integral over $S$. Then there is a well defined group homomorphism $\phi: C l(S) \rightarrow C l(T)$. ([7], theorem (6.2), page 20).

Theorem 2. Let $T$ be a Krull domain of characteristic $p \neq 0$ and $\mathcal{G}$ a finite group of derivation of $T$. Let $S \subset T$ be the fixed subring of $\mathcal{G}$ and let $L$ denote the quotient field of $T$. Let $\Delta_{1}, \cdots, \Delta_{m}$ be a basis of $\mathcal{G}$ over $Z / p Z$. Then $S$ is a Krull domain, $T$ is integral over $S$, and the kernel of the homomorphism $\phi: C l(S)$ $\rightarrow C l(T)$ described in (1) is isomorphic to a subgroup of $V_{0} / V_{0}^{\prime}$ where $V_{0}$ and $V_{0}^{\prime}$ are the following additive subgroups of $L^{m}: V_{0}=\left\{\left(t^{-1} \Delta_{1} t, \cdots, t^{-1} \Delta_{m} t\right): t \in L\right.$ and $t^{-1} \Delta_{\imath} t \in T$ for $\left.1 \leqq i \leqq m\right\}$ and $V_{0}^{\prime}=\left\{\left(u^{-1} \Delta_{1} u, \cdots, u^{-1} \Delta_{m} u\right): u\right.$ is a unit in $\left.T\right\}$. ([2], Corollary 17.3, page 92).

Remark 3. If $I$ is a divisorial ideal of $S$ whose class group is in the kernel of $C l(S) \rightarrow C l(T)$, then $T:(T: I T)$ is a principal ideal, say $t T$, for some $t \in T$. The injection $\operatorname{ker} \phi \rightarrow V_{0} / V_{0}^{\prime}$ maps $I$ to $\left(t^{-1} \Delta_{1} t, \cdots, t^{-1} \Delta_{m} t\right)$.

Theorem 4. Let $T[G]$ denote the $S$-subalgebra of $\operatorname{End}_{s}(T)$ generated by $T$ and $\mathcal{G}$. If $T[\mathcal{G}]=\operatorname{End}_{s}(T)$, then $\operatorname{ker} \psi \rightarrow V_{0} / V_{0}^{\prime}$ is an isomorphism. ([2], theorem 17.4, page 93).

Proposition 5. Let $L^{\prime}$ be the quotient field of $S$. If $\left[L: L^{\prime}\right]=p$ and $D(T)$ is contained in no height one prime ideal of $T$, then $\operatorname{ker} \psi \rightarrow V_{0} / V_{0}^{\prime}$ is an isomorphism. ([7], Theorem 2.1, page 62).

Proposition 6. Let 1 be a graded Krull domain. Let $\operatorname{Div}_{h}(.4)$ denote the subgroup of $\operatorname{Div}(A)$ generated by the homogeneous divisorial (prime) ideals and set $\operatorname{Prin}_{h}(A)=\operatorname{Prin}(A) \cap \operatorname{Div}_{h}(A)$. Then the inclusion $\operatorname{Div}_{h}(A) \rightarrow \operatorname{Div}(A)$ induces a bijection

$$
\operatorname{Div}_{h} A / \operatorname{Prin}_{h}(A) \longrightarrow C l(A) \text {. }
$$

([2], proposition 10.2 , page 42 ).

Proposition 7. In (2) assume $T=T_{0} \oplus T_{1} \oplus T_{2} \oplus \cdots$ is a graded ring and $\Delta_{2}$, $1 \leqq i \leqq m$, are graded derivations of degree $e_{\imath}$ (i.e. $\Delta_{\imath}\left(T_{j}\right) \subset T_{j+e_{i}}$ for each $\left.j\right)$. For each $j=0,1, \cdots$, let $S_{j}=T_{j} \cap\left(\cap_{G} \Delta^{-1}(0)\right)$. Assume $S_{0}=T_{0}$. Then $V_{0}^{\prime}=0$ and $S$ is the graded subring of $T, S=S_{0} \oplus S_{1} \oplus \cdots$. If , in addition, $T$ is a unique factorization domain, then $C l(S)=\operatorname{ker} \psi$ and the image of the mapping $\operatorname{ker} \psi \rightarrow V_{0}$ is spanned by $m$-tuples $\left(t^{-1} \Delta_{1} t, \cdots, t^{-1} \Delta_{m} t\right) \in T^{m}$, where $t$ is homogeneous irreducible in $T$.

Proof. If $u$ is a unit in $T, u \in T_{0}$. Since $S_{0}=T_{0}, V_{0}^{\prime}=0$. Given $j=0,1, \cdots, T_{\text {, }}$ $\cap\left(\cap \Delta_{\underline{G}}^{-1}(0)\right) \subset T_{j} \cap\left(\bigcap_{i=1}^{m} \Delta_{i}^{-1}(0)\right)$. The reverse inclusion holds since the $\Delta_{i}$ span $\mathcal{G}$. Therefore $S_{j}=T_{j} \cap\left(\bigcap_{\imath=1}^{m} \Delta_{i}^{-1}(0)\right)$. Since the $\Delta_{\imath}$ are homogeneous, an element 
$t \in \bigcap_{i=1}^{m} \Delta_{i}^{-1}(0)$ if and only if its homogeneous parts belong to $\bigcap_{i=1}^{m} \Delta_{i}^{-1}(0)$. Thus $S=S_{0} \oplus S_{1} \oplus \cdots$.

If $T$ is a unique factorization domain, then $C l(T)=0$ and obviously $C l(S)=$ $\operatorname{ker} \psi$. By (2), (3) and (6), the image of $\operatorname{ker} \psi$ in $V_{0}$ will be generated by $m$ tuples $\left(t^{-1} \Delta_{1} t, \cdots, t^{-1} \Delta_{m} t\right) \in T^{m}$ where $t$ is a homogeneous element of $T$. $t$ can be factored as a product $t=v_{1}^{n_{1}} \cdots v_{s}^{n_{s}}$, where the $w_{\imath}$ are irreducible homogeneous elements in $T$ and the $n_{i}$ are positive integers. If one of the $n_{\imath}=0(\bmod p)$, then $t$ can be replaced by $w_{i}^{-n_{i}}$; so we may assume no $n_{\imath}=0(\bmod p)$.

If for some $j, t^{-1} \Delta_{j} t \neq 0$, then $t^{-1} \Delta_{j} t=\sum_{i} n_{\imath} w_{i}^{-1} \Delta_{j}\left(w_{\imath}\right) . \quad t^{-1} \Delta_{j} t \in T$ implies $\sum_{i} n_{\imath} \frac{w_{1} \cdots w_{s}}{w_{i}} \Delta_{j}\left(w_{\imath}\right) \in w_{1} \cdots w_{s} T$. Since the $w_{\imath}$ are pairwise coprime, $w_{\imath}$ must divide $\Delta_{j}\left(w_{\imath}\right)$ in $T$. Thus $w_{i}^{-1} \Delta_{j}\left(w_{\imath}\right) \in T$ for each $i$ and $j$ and $\left(t^{-1} \Delta_{1} t, \cdots, t^{-1} \Delta_{m} t\right)$ $=\sum_{i} n_{\imath}\left(w_{i}^{-1} \Delta_{1} w_{i}, \cdots, w_{i}^{-1} \Delta_{m} w_{\imath}\right)$.

Definition 8. Let $K$ be a field of characteristic $p \neq 0$. A set $\mathscr{D}$ of derivations of $K$ is called a restricted $K$-Lie algebra of derivations of $K$ if: (1) $\mathscr{D}$ is closed under addition: (2) $\mathscr{D}$ is closed under bracket product (3) $\mathscr{D}$ is closed under $p$-th powers; (4) $\mathscr{D}$ is closed under multiplication by elements of $K$.

Theorem 9 (Jacobson). Let $K$ be a field of characteristic $p \neq 0$. Let $\mathscr{D}$ be a restricted $K$-Lie algebra of derivations of $K$ such that $[\mathscr{D}: K]=m<\infty$. Then: (1) If $K^{\prime}$ is the subfield of $\mathscr{D}$ constants, then $K$ is purely inseparable of exponent $\leqq 1$ over $K^{\prime}$ and $\left[K: K^{\prime}\right]=p^{m}$; (2) if $\mathscr{D}$ is any derivation of $K$ over $K^{\prime}$, then $D \in \mathscr{D}$; (3) if $\left(D_{1}, \cdots, D_{m}\right)$ is any basis for $\mathscr{D}$ over $K$, then the set of monomials

$$
D_{1}^{k_{1}} \cdots D_{m}^{k_{m}} ; 0 \leqq k_{i}<p, \quad\left(D_{i}^{0}=1\right)
$$

is a basis for the ring $\operatorname{End}_{K^{\prime}}(K)$ considered as a vector space over $K$. ([4], theorem 19, page 186).

Lemma 10. Let $K$ be a perfect field of characteristic $p \neq 0$. Let $B$ be $a$ finitely generated K-integral domain of dimension $d$. Let $C=B^{p}$, the ring of $p$-th powers of elements of $B$. Then the degree of $B$ over $C$ is $p^{d}$.

Proof. $B=K\left[w_{1}, \cdots, w_{t}\right]$ for some $w_{i} \in B$. Then $C=K\left[w_{1}^{p}, \cdots, w_{t}^{p}\right]$. By Noether's normalization theorem, there exists $y_{1}, \cdots, y_{d} \in B$ such that $B$ is separable algebraic over $K\left[y_{1}, \cdots, y_{d}\right]$ and $y_{1}, \cdots, y_{d}$ are algebraically independent over $K$. Let $L_{B}, L_{C}$ be the fields of quotients of $B, C$, respectively. Clearly, $\left[L_{B}: K\left(y_{1}, \cdots, y_{d}\right)\right]=\left[L_{C}: K\left(y_{1}^{p}, \cdots, y_{d}^{p}\right)\right]$ and the result follows.

\section{§ 2. Purely Inseparable Extensions of Complete Intersections}

1. Let $k$ be an algebraically closed field of characteristic $p \neq 0$ and let $k^{[n]}$ denote the polynomial ring in $n$ variables over $k$. Assume $h_{1}, \cdots, h_{r}(r \leqq n-2)$ 
are homogeneous polynomials in $k^{[n]}$ such that the ideal $P$ they generate is a height $r$ prime ideal in $k^{[n]}$. Let $R=k^{[n]} / P$ and for $f \in k^{[n]}$ denote its image in $R$ by $\bar{f}$. For each integer $m \geqq 0$, let $R_{m}=k\left[\bar{x}_{1}^{p^{m}}, \cdots, \bar{x}_{n}^{p^{m}}\right] \subset R$. $R_{m}$ is a subring of $R$ and is ring isomorphic (not $k$-isomorphic) to $R$ since $k$ is perfect.

Throughout assume $R$ is a unique factorization domain. Also assume $h_{r+1}$ $\in k^{[n]}$ is homogeneous such that $\operatorname{deg}\left(h_{r+1}\right) \neq 0(\bmod p)$ and $h_{r+1}$ factors as a product $\bar{h}_{r+1}=\bar{u}_{1} \bar{u}_{2} \cdots \bar{u}_{q}$ of $q$ distinct irreducible elements in $R$. Note that this last assumption implies $\bar{h}_{r+1} \notin R_{1}$.

For each integer $m \geqq 0$, let $S_{m}=R_{m}\left[\bar{h}_{r+1}\right]$ and $X_{m} \subset A_{k}^{n+1}$ be the variety defined by the equations $h_{1}=\cdots=h_{r}=x_{n+1}^{p^{m}}-h_{r+1}=0$.

Lemma 2. For each $m$, the coordinate ring of $X_{m}$ is isomorphic $S_{m}$.

Proof. Let $\phi: k\left[x_{1}, \cdots, x_{n+1}\right] \rightarrow S_{m}$ be the surjection that sends $x_{\imath}$ to $\bar{x}_{7}^{p^{m}}$ for $1 \leqq i \leqq n, \alpha$ to $\alpha^{p^{m}}$ for $\alpha \in k$ and $x_{n+1}$ to $\bar{h}_{r+1}$. Let $Q \subset k^{[n+1]}$ be the ideal generated by $h_{1}, \cdots, h_{r}, x_{n+1}^{p^{m}}-h_{r+1}$. Since $\bar{h}_{r+1} \notin R_{1}, Q$ is a prime ideal of height $r+1$ contained in $\operatorname{ker} \phi$. We have $\operatorname{dim}\left(R_{m}\left[\bar{h}_{r+1}\right]\right)=\operatorname{dim}(R)=n-r$. Thus $\operatorname{ker} \phi$ is a height $r+1$ prime ideal and $\operatorname{ker} \phi=Q$. Therefore $k^{[n+1]} / Q$ is isomorphic to $S_{m}$.

3. For each integer $m \geqq 0$, let $S_{m}^{\prime}$ be the ring of $p$-th powers of elements of $S_{m} . S_{m}^{\prime}$ and $S_{m}$ are isomorphic and $S_{m}^{\prime} \subset S_{m+1} \subset S_{m}$. Denote by $E_{m}, F_{m}$ and $F_{m}^{\prime}$ the quotient fields of $R_{m}, S_{m}$ and $S_{m}^{\prime}$, respectively.

Lemma 4。 For each $m \geqq 0$ : (i) $\left[E_{m}: E_{m+1}\right]=\left[F_{m}: F_{m}^{\prime}\right]=p^{n-r}$; (ii) $\left[F_{m+1}: F_{m}^{\prime}\right]$ $=p$; (iii) $\left[F_{m}: F_{m+1}\right]=p^{n-r-1}$; (iv) $\left[F_{m}: E_{m}\right]=p^{m+1}$.

Proof. (i) is an immediate consequence of Lemma (1.10). (ii) is obvious. (iii) follows from (i) and (ii). To prove (iv), note that $F_{m}=E_{m}\left(\bar{h}_{r+1}\right)$. Then $\left.\left[F_{m}: E_{m}\right]=\prod_{i=0}^{m-1}\left[E_{m}\left(\bar{h}_{r+1}^{p^{i}}\right)\right]: E_{m}\left(\bar{h}_{r+1}^{p^{i+1}}\right)\right] . E_{m}\left(\bar{h}_{r+1}^{p^{i}}\right) \cong E_{m-\imath}\left(\bar{h}_{r+1}\right)=F_{m-i}$, and $E_{m}\left(\bar{h}_{r+1}^{p^{i+1}}\right)$ $\cong E_{m-\imath}\left(\bar{h}_{r+1}^{p}\right)=F_{m-\imath-1}^{\prime}$, where the two isomorphisms are nothing other than the operation of taking $p^{i}$-th roots. Thus $\left[F_{m}: E_{m}\right]=\prod_{i=0}^{m-1}\left[F_{m-\imath}: F_{m-\imath-1}^{\prime}\right]=p^{m}$ by (ii).

5. From here on assume that $X_{1}$ is regular in codimension one. Then $X_{m}$ is regular in codimension one for each $m$ and $S_{m}$ is noetherian integrally closed; hence $S_{m}$ is a Krull domain. By (1.1) and (1.3) we obtain well defined group homomorphisms $\phi_{m}^{\prime}: C l\left(S_{m}^{\prime}\right) \rightarrow C l\left(S_{m+1}\right)$ and $\phi_{m}: C l\left(S_{m+1}\right) \rightarrow C l\left(S_{m}\right)$, which we'll study via (1.3).

Definition 6. If $A$ is a ring and $f_{1}, \cdots, f_{s} \in A\left[x_{1}, \cdots, x_{s}\right]$, the polynomial ring in $s$ variables over $A$, let $\partial\left(f_{1}, \cdots, f_{s}\right) / \partial\left(x_{1}, \cdots, x_{s}\right)$ denote the determinant 
of the Jacobian matrix $\left[\partial f_{i} / \partial x_{j}\right]$.

\section{$\S 3$. The Calculation of $\operatorname{ker} \phi_{m}^{\prime}$}

1. For each $(r+1)$-tuple $I=\left(i_{1}, \cdots, i_{r+1}\right)$ of integers with $1 \leqq i_{j} \leqq n$, let $D_{I}$ be the derivation on $k\left(x_{1}, \cdots, x_{n}\right)$ defined by $D_{I}=\partial\left(, h_{1}, \cdots, h_{r}\right) / \partial\left(x_{\imath_{1}}, \cdots, x_{\imath_{r+1}}\right)$. Since $D_{I}\left(h_{i}\right)=0$ for each $i=1, \cdots, r ; D_{I}$ induces a derivation on $E_{0}=k\left(\bar{x}_{1}, \cdots, \bar{x}_{m}\right)$. We will also denote this derivation by $D_{I}$; it should be clear from the context which one is meant.

Lemma 2. $R \cap\left(\bigcap_{I} D_{I}^{-1}(0)\right)=R_{1}$.

Proof. Since $R$ is factorial, $R$ is regular in codimension one. Thus the maximal minors of the Jacobian matrix $\left[\partial h_{\imath} / \partial x_{j}\right]_{1 \leqq i \leqq r}$ have greatest common divisor 1 in $R$. In particular, at least one maximal minor (actually at least two) has nonzero image in $R$. Without loss of generality we may assume $\partial\left(h_{1}, \cdots, h_{r}\right) /$ $\partial\left(x_{1}, \cdots, x_{r}\right)$ has nonzero image in $R$.

For each $s=r+1, \cdots, n$, let $D_{s}=D_{(1, \cdots, r, s)}$. Then $E_{0} \supset E_{0} \cap D_{r+1}^{-1}(0) \supset E_{0} \cap$ $\left(\bigcap_{s=r+1}^{r_{+}+2} D_{s}^{-1}(0)\right) \supset \cdots \supset E_{0} \cap\left(\bigcap_{s=r+1}^{n} D_{s}^{-1}(0)\right) . \quad$ Each containment is proper since $x_{t} \in$ $\bigcap_{s<t} D_{s}^{-1}(0)$ and $D_{t}\left(\bar{x}_{t}\right) \neq 0$. Also, $E_{1} \subset E_{0} \cap\left(\bigcap_{I} D_{I}^{-1}(0)\right) \subset E_{0} \cap\left(\bigcap_{s=r+1}^{n} D_{s}^{-1}(0)\right)$. By $(2.4)$, $\left[E_{0}: E_{1}\right]=p^{n-r}$, which forces $E_{1}=E_{0} \cap\left(\cap_{I} D_{I}^{-1}(0)\right)$. Thus $R_{1}$ and $R \cap\left(\cap_{I} D_{I}^{-1}(0)\right)$ have the same quotient field. Since $R_{1}$ is integrally closed, $R_{1}=R \cap\left(\cap_{I} D_{I}^{-1}(0)\right)$.

Remark 3. By (1.1), $\bar{h}_{r+1} \notin R_{1}$. By (3.2), there exists an $(r+1)$-tuple $I_{0}$ such that $D_{I_{0}}\left(\bar{h}_{r+1}\right) \neq 0$. Let $\beta=D_{I_{0}}\left(\bar{h}_{r+1}\right)$. For each integer $m \geqq 0$, let $\Delta_{m}$ be the restriction of the derivation $\beta^{-1} D_{I_{0}}$ on $E_{0}$ to $S_{m}$.

Lemma 4. $\Delta_{m}$ maps $S_{m}$ into $S_{m}$ and has kernel $S_{m-1}^{\prime}$.

Proof. Let $\alpha \in S_{m}$. By (2.4), $\alpha=\sum_{i=0}^{p-1} \alpha_{\imath} \bar{h}_{r+1}^{i}$ for unique $\alpha_{\imath} \in S_{m-1}^{\prime}$. Then $\Delta_{m}(\alpha)$ $=\sum_{i=0}^{p-1} i \alpha_{i} \bar{h}_{r+1}^{i-1}$. Thus $\Delta_{m}(\alpha) \in S_{m}$ and $\Delta_{m}(\alpha)=0$ if and only if $\alpha_{\imath}=0$ for $1 \leqq i \leqq p-1$; that is, if and only if $\alpha \in S_{m-1}^{\prime}$.

Proposition 5. The mapping $\phi_{m}^{\prime}: \operatorname{Cl}\left(S_{m}^{\prime}\right) \rightarrow C l\left(S_{m+1}\right)$ described in (2.5) is an injection.

Proof. $\Delta_{m}\left(\bar{h}_{r+1}\right)=1$ and $\left[F_{m+1}: F_{m}^{\prime}\right]=p . \quad$ By (2.5), (1.7), and (3.4), $\operatorname{ker} \phi_{m}^{\prime}$ is isomorphic to $V_{0}$, where $V_{0}$ is spanned by the logarithmic derivatives $t^{-1} \Delta_{m} t \in$ $S_{m+1}$, where $t \in S_{m+1}$ is homogeneous with respect to the grading $S_{m+1}$ inherits from $R . t$ homogeneous and $\operatorname{deg}\left(\bar{h}_{r+1}\right) \neq 0(\bmod p)$ implies $t=\alpha \bar{h}_{r+1}^{j}$ for some 
$\alpha \in S_{m}^{\prime}$ and integer $j, 0 \leqq j \leqq p-1$. If $t^{-1} \Delta_{m} t \in S_{m_{+1}}$, then either $j=0$ or $\bar{h}_{r+1}^{-1} \in$ $S_{m+1}$. Thus $t^{-1} \Delta_{m} t=0$ and $V_{0}=0$.

Proposition 6. Let $\mathcal{Q}$ denote the vector space over $E_{0}$ generated by the $D_{I}$ defined in (3.1). Then if $D$ is a derivation on $E_{0}, D \in \mathcal{G}$.

Proof. Let $\mathscr{D}$ denote the vector space of derivations on $E_{0}$. The map $D \rightarrow$ $\left(D \bar{x}_{1}, \cdots, D \bar{x}_{n}\right)$ is a $E_{0}$-vector space monomorphism from $\mathscr{D}$ to $E_{0}^{n}$. If $D \in \mathscr{D}$, $0=D\left(\bar{h}_{\imath}\right)=\sum_{i} \frac{\overline{\partial h_{2}}}{\partial x_{j}} D \bar{x}_{j}$. Thus $D \in \mathscr{D}$ implies $\left[\frac{\overline{\partial h_{i}}}{\partial x_{j}}\right] D(\bar{x})=0$. Since $R$ is factorial, $R$ is regular in codimension 1 , which implies the rank of $\left[\frac{\overline{\partial h_{i}}}{\partial x_{j}}\right]$ is $r$. Therefore $D$ is of dimension at most $n-r$ over $E_{0}$.

Assuming again that $\partial\left(h_{1}, \cdots, h_{r}\right) / \partial\left(x_{1}, \cdots, x_{r}\right)$ has nonzero image in $R$, we get that the column matrix $\left[D_{s}\right]_{r+1 \leqq s \leqq n}$ (where $D_{s}$ is defined in the proof of (3.2)) is mapped to an $(n-r) \times n$ matrix under $\mathscr{D} \rightarrow E_{0}^{n}$ that contains an $(n-r) \times(n-r)$ nonzero scalar submatrix $\overline{\partial\left(h_{1}, \cdots, h_{r}\right) / \partial\left(x_{1}, \cdots, x_{r}\right)} \cdot I_{n-r}$. Thus $\mathcal{G}$ has dimension at least $n-r$ over $E_{0}$, which shows that $\mathscr{D}=\mathcal{G}$.

Corollary 7. Let $\mathcal{G}$ denote the vector space over $E_{0}$ generated by the $D_{I}$. Then $\mathcal{G}$ is a restricted Lie algebra of derivations over $E_{0}$. Furthermore, $R[\mathcal{G}]=$ $\operatorname{End}_{R_{1}}(R)$.

Proof. $\mathscr{D}$, the space of derivations on $E_{0}$, is a restricted Lie algebra of derivations over $E_{0}$. By (3.6), $\mathscr{D}=\mathcal{G}$. Both $R[\mathcal{G}]$ and $\operatorname{End}_{R_{1}}(R)$ are locally free $R_{1}$ modules ([1], page 86 , exercise 16 and page 99 , exercise 5 ). By (1.9) and a rank argument $R[\mathcal{G}]=\operatorname{End}_{R_{1}}(R)$.

Corollary 8. Let $t \in E_{0}$. If $t^{-1} D_{I} t \in R$ for all $I$, then $D_{I}(t)=0$ for all $I$.

Proof. Let $W=\left\{\left(t^{-1} D_{I} t\right): t \in E_{0}\right.$ and $t^{-1} D_{I} t \in R$ for all $\left.I\right\}$. Since the units of $R$ are the nonzero elements of $k$, by (1.2), (1.4), (3.2) and (3.7), $W=0$.

\section{$\S 4$. The Calculation of $C l\left(S_{1}\right)$}

1. For each $(r+2)$-tuple $J=\left(i_{1}, \cdots, i_{r+2}\right)$ of integers with $1 \leqq i_{j} \leqq n$, let $D_{J}$ be the derivation on $k\left(x_{1}, \cdots, x_{n}\right)$ defined by $D_{J}=\partial\left(, h_{1}, \cdots, h_{r+1}\right) / \partial\left(x_{i_{1}}, \cdots, x_{\imath_{r+2}}\right)$. Since $D_{J}\left(h_{\imath}\right)=0$ for $1 \leqq i \leqq r, D_{J}$ induces a derivation on $E_{0}$, which we will also denote by $D_{J}$.

Lemma 2. $R \cap\left(\bigcap_{J} D_{J}^{-1}(0)\right)=S_{1}$.

Proof. Similar to (3.2).

Proposition 3. Let $W_{0}$ be the additive group generated by $\left\{\left(t^{-1} D_{J} t\right): t \in R\right.$ is 
irreducible and homogeneous and $t^{-1} D_{J} t \in R$ for each $\left.J\right\}$. Then $C l\left(S_{1}\right)$ is isomorphic to a subgroup of $W_{0}$.

Proof. Follows from (1.7) and (4.2).

4. Recall that $\bar{h}_{r+1}=\bar{u}_{1} \cdots \bar{u}_{q}$ is a product of $q$ distinct irreducible elements in $R$ and $\operatorname{deg}\left(h_{r+1}\right) \neq 0(\bmod p)$.

Lemma 5. For each $i=1, \cdots, q-1$, let $Q_{i}$ be the height one prime ideal in $S_{1}$ generated by $x_{n+1}$ and $u_{i}$. Then the classes of the $Q_{i}$ generate a subgroup of $\mathrm{Cl}\left(S_{1}\right)$ of order $p^{q-1}$.

Proof. By (4.3), $C l\left(S_{1}\right) \rightarrow W_{0}$ is an injection. By (1.3), $Q_{i}$ maps to $\left(\bar{u}_{i}^{-1} D_{J}\left(\bar{u}_{i}\right)\right)$ in $W_{0}$.

Claim. The elements $\left(\bar{u}_{i}^{-1} D_{J}\left(\bar{u}_{i}\right)\right), 1 \leqq i \leqq q-1$, are $F_{p}$-independent; $F_{p}$ the prime subfield of $k$.

Suppose $e_{\imath} \in F_{p}, 1 \leqq i \leqq q-1$, such that $\sum_{i} e_{2}\left(\bar{u}_{i}^{-1} D_{J} \bar{u}_{i}\right)=0$. Let $H=\prod_{i} u_{i}^{e_{i}}$. Then $D_{J}(\bar{H})=0$ for each $J$, which implies by (4.2) that $E_{1} \subset E_{1}(\bar{H}) \subset F_{1}=E_{1}\left(\bar{h}_{r+1}\right)$. If $\bar{H} \notin E_{1}$, then $E_{1}(\bar{H})=E_{1}\left(\bar{h}_{r+1}\right)$, which implies there exists $\alpha_{j} \in k^{[n]}$ such that $\bar{\alpha}_{p}^{p} \bar{H}=\sum_{j=0}^{p-1} \bar{\alpha}_{j}^{p} \bar{h}_{r+1}^{j}$. Since $H$ and $h_{r+1}$ are homogeneous elements and $P$ a homogeneous ideal in $k^{[n]}$, we may assume that the $\alpha_{j}$ are homogeneous polynomials as well. Since $\operatorname{deg}\left(\alpha_{j}^{p} h_{r+1}^{j}\right)=j\left(\operatorname{deg}\left(h_{r+1}\right)\right)(\bmod p)$ and $\operatorname{deg}\left(h_{r+1}\right) \neq 0(\bmod p)$, it follows $\bar{\alpha}_{0}^{p} \bar{H}=\bar{\alpha}_{j_{0}}^{p} \bar{h}_{r+1}^{j_{0}}$ for some $j_{0}=0,1, \cdots, p-1$. If $j_{0} \neq 0$, then this implies $\bar{u}_{q} \in E_{1}$, which contradicts the irreducibility of $\bar{u}_{q}$ in $R$. If $j_{0}=0$, then $\bar{H}$ must in fact belong to $E_{1}$. But if $\bar{H} \in E_{1}$, then each $e_{i}=0(\bmod p)$. This proves the claim and hence the lemma.

Theorem 6. $C l\left(S_{1}\right)$ is a direct sum of $q-1$ copies of $Z / p Z$.

Proof. By (4.3) and (4.5) it is enough to show that $W_{0}$ has order at most $p^{q-1}$. Let $\bar{u} \in R$ be irreducible homogeneous such that $\bar{w}_{J}=\bar{u}^{-1} D_{J}(\bar{u}) \in R$ for each $J$. Then $\bar{\imath}$ divides the images in $R$ of all of the maximal minors of the matrix

$$
M=\left|\begin{array}{cccc}
\frac{\partial u}{\partial x_{1}} & \frac{\partial u}{\partial x_{2}} & \cdots & \frac{\partial u}{\partial x_{n}} \\
\frac{\partial h_{1}}{\partial x_{1}} & \frac{\partial h_{1}}{\partial x_{2}} & \cdots & \frac{\partial h_{1}}{\partial x_{n}} \\
\cdots & \cdots & \cdots & \cdots \\
\frac{\partial h_{r+1}}{\partial x_{1}} & \frac{\partial h_{r+1}}{\partial x_{2}} & \cdots & \frac{\partial h_{r+1}}{\partial x_{n}}
\end{array}\right|
$$

By Euler's formula, $\bar{u}$ divides in $R$ the images of the maximal minors of the 
matrix obtained from $M$ by replacing the $j$-th column by the $(r+2) \times 1$ vector

$$
\left[\begin{array}{c}
d \cdot u \\
0 \\
\vdots \\
0 \\
d^{\prime} \cdot h_{r+1}
\end{array}\right]
$$

where $d=\operatorname{deg}(u)$ and $d^{\prime}=\operatorname{deg}\left(h_{r_{+1}}\right)$. Therefore $\bar{u}$ is a factor of $\bar{h}_{r+1}$ or $\bar{u}$ is a common factor of the images in $R$ of each of the maximal minors of the matrix

$$
\left[\begin{array}{cccc}
\frac{\partial u}{\partial x_{1}} & \frac{\partial u}{\partial x_{2}} & \cdots & \frac{\partial u}{\partial x_{n}} \\
\frac{\partial h_{1}}{\partial x_{1}} & \frac{\partial h_{1}}{\partial x_{2}} & \cdots & \frac{\partial h_{1}}{\partial x_{n}} \\
\cdots & \cdots & \cdots & \cdots \\
\frac{\partial h_{r}}{\partial x_{1}} & \frac{\partial h_{r}}{\partial x_{2}} & \cdots & \frac{\partial h_{r}}{\partial x_{n}}
\end{array}\right] .
$$

The latter implies $\bar{u}^{-1} D_{I} \bar{u} \in R$ for each of the derivations $D_{I}$ described in (3.1), which by (3.2) and (3.8) implies $\bar{u} \in R_{1}$. Since $\bar{u}$ is irreducible in $R$, it must be that $\bar{u}$ is a $k^{*}$ multiple of one of the $\bar{u}_{i}$. By (4.3) we get $W_{0}$ is generated by $\left(\bar{u}_{i}^{-1} D_{J} \bar{u}_{i}\right), 1 \leqq i \leqq q$. We have for each $J, \sum_{i=1}^{q} \bar{u}_{i}^{-1} D_{J} \bar{u}_{i}=\bar{h}_{r+1}^{-1} D_{J}\left(\bar{h}_{r+1}\right)=0$. Therefore $W_{0}$ is generated by $\left(\bar{u}_{i}^{-1} D_{J} \bar{u}_{i}\right), 1 \leqq i \leqq q-1$. Since $W_{0}$ is a $p$-group, the order of $W_{0}$ is at most $p^{q-1}$.

Corollary 7. $C l\left(X_{1}\right)$ is generated by the codimension one cycles on $X_{1}$ defined by $u_{i}=0,1 \leqq i \leqq q-1$.

Proof. By the proof of (4.5) and by theorem (4.6).

\section{$\S 5$. The Calculation of $C l\left(S_{m}\right)$}

1. Given $\alpha \in F_{m}$, by (2.4) there exist unique $\alpha_{\imath} \in E_{0}$, such that $\alpha=\sum_{i=0}^{m-1} \alpha_{i}^{p m} \bar{h}_{r+1}^{i}$. For each derivation $D_{J}$ defined in (4.1) and each integer $m \geqq 0$, define a mapping $D_{J}^{(m)}$ on $F_{m}$ by the formula

$$
D_{J}^{(m)}(\alpha)=\sum_{i=0}^{m-1}\left(D_{J}\left(\alpha_{i}\right)\right)^{p^{m}} \bar{h}_{r+1}^{i} .
$$

Lemma 2. $D_{J}^{(m)}$ is a derivation on $F_{m}$.

Proof. $D_{J}^{(m)}$ is clearly additive. Given $\alpha=\sum_{i=0}^{p m-1} \alpha_{i}^{p^{m}} \bar{h}_{r+1}^{i}$ and $\beta=\sum_{i=0}^{p m-1} \beta_{i}^{p^{m}} \bar{h}_{r+1}^{i}$ 
in $F_{m}$, we must show that $D_{J}^{(m)}(\alpha \beta)=\alpha D_{J}^{(m)} \beta+\beta D_{J}^{(m)} \alpha$. We argue by induction on the number of nonzero coefficients appearing in $\alpha$ plus the number of nonzero coefficients appearing in $\beta$.

Suppose this sum is 2. Then $\alpha=\alpha_{i}^{p m} \bar{h}_{r+1}^{i}$ and $\beta=\beta_{j}^{p m} \bar{h}_{r+1}^{j}$ for some $\alpha_{i}, \beta_{j}$ $\in E_{0}$ and nonnegative integers $i$ and $j$. Then $D_{J}^{(m)}(\alpha \beta)=D_{J}^{(m)}\left(\alpha_{i} \beta_{j}\right)^{p^{m}} h_{r+1}^{i+j}=$ $\left(D_{J}\left(\alpha_{i} \beta_{j}\right)\right)^{p^{m}} \bar{h}_{r+1}^{i+j}=\left(\alpha_{2} D_{J} \beta_{j}+\beta_{j} D_{J} \alpha_{\imath}\right)^{p^{m}} \bar{h}_{r+1}^{i+j}=\alpha_{i}^{p^{m}} \bar{h}_{r+1}^{i} D_{J}^{(m)}\left(\beta_{j}^{p^{m}} \bar{h}_{r+1}^{j}\right)+$ $\beta_{j}^{p^{m}} \bar{h}_{r+1}^{j} D_{J}^{(m)}\left(\alpha_{i}^{p^{m}} \bar{h}_{r+1}^{i}\right)=\alpha D_{J}^{(m)} \beta+\beta D_{J}^{(m)} \alpha$.

Now assume that the total number of nonzero coefficients appearing in $\alpha$ and $\beta$ is greater than 2. Let $j_{0}$ be the highest power of $\bar{h}_{r+1}$ with nonzero coefficient in $\beta$. Then $D_{J}^{(m)}(\alpha \beta)=D_{J}^{(m)}\left(\alpha\left(\beta-\beta_{j_{0}}^{p^{m}} \bar{h}_{r+1}^{j_{0}}\right)\right)+D_{J}^{(m)}\left(\alpha \beta_{j_{0}}^{p^{m}} \bar{h}_{r+1}^{j_{0}}\right)$, which by the induction hypothesis and the additivity of $D_{J}^{(m)}$, equals to $\alpha D_{J}^{(m)} \beta+$ $\beta D_{J}^{(m)} \alpha$.

Lemma 3. $S_{n+1}$ is the fixed subring of the derivations $D_{J}^{(m)}$ acting on $S_{m}$.

Proof. Given $\alpha \in S_{m}, \alpha=\sum \alpha_{\imath}^{p^{m}} \bar{h}_{r+1}^{i}, \alpha_{\imath} \in R . \quad D_{J}^{(m)}(\alpha)=0$ for each $J$ if and only if $D_{J}\left(\alpha_{i}\right)=0$ for each $J$ and $0 \leqq i \leqq p^{m}-1$. By (4.2) we get $D_{J}^{(m)}(\alpha)=0$ for each $J$ implies each $\alpha_{\imath} \in S_{1}$ and hence $\alpha \in S_{m+1}$.

Definition 4. For each integer $m \geqq 1$, let $W_{m}$ be the additive group generated by $\left\{\left(t^{-1} D_{J}^{(m)} t\right): t\right.$ is a homogeneous element in $S_{m}$ and $t^{-1} D_{J}^{(m)} t \in S_{m}$ for each $\left.J\right\}$.

Lemma 5. If $\left(u_{J}\right) \subseteq W_{0}$ then $\left(u_{J}^{p^{m}}\right) \equiv W_{m}$ and the mapping $\left(u_{J}\right) \rightarrow\left(u_{J}^{p^{m}}\right)$ from $W_{0}$ to $W_{m}$ is an isomorphism.

Proof. If $t \equiv E_{0}$ and $t^{-1} D_{J} t=u_{J} \fallingdotseq R$, then $t^{p^{m}} \in F_{m}$ and $t^{-p^{m}} D_{J}^{(m)}\left(t^{p m}\right)=$ $\left(t^{-1} D_{J} t\right)^{p^{m}}=u_{J}^{p^{m}} \equiv R_{m} \subset S_{m}$. This also shows that $\left(u_{J}\right) \rightarrow\left(u_{J}^{p^{m}}\right)$ defines an injection from $W_{0}$ to $W_{m}$.

Suppose $t^{\prime} \subseteq S_{m}$ is homogeneous. Since $\operatorname{deg}\left(h_{r+1}\right) \neq 0(\bmod p), t^{\prime}=\bar{\alpha}^{p^{m}} \bar{h}_{r+1}^{i}$, where $\alpha \equiv k^{[n]}$ is homogeneous and $i$ is a nonnegative integer. If $\left(t^{\prime}\right)^{-1} D_{J}^{(m)}\left(t^{\prime}\right)$ is a nonzero element of $S_{m}$, then $\left(t^{\prime}\right)^{-1} D_{J}^{(m)}\left(t^{\prime}\right)=\left(\alpha^{-1} D_{J} \alpha\right)^{p^{m}} \in S_{m} \cap E_{m}=R_{m}$. Thus $\alpha^{-1} D_{J} \alpha \cong R$. Therefore $W_{0} \rightarrow W_{m}$ is also a surjection.

Theorem 6. $C l\left(S_{m}\right)$ is a direct sum of $q-1$ copies of $Z / p^{m} Z$, generated by the height one primes $Q_{i}^{(m)}=\bar{h}_{r+1} S_{m}+\bar{u}_{i}^{p^{m}} S_{m}$ in $S_{m}, 1 \leqq i \leqq q-1$.

Proof. For each $i=1, \cdots, q-1$ and positive integer $m$, let $P_{i}^{(m)}=\bar{h}_{r+1}^{p} S_{m}^{\prime}+$ $\bar{\imath}_{i}^{p}{ }^{m+1} S_{m}^{\prime}$. Then $Q_{i}^{(m)} \cap S_{m+1}=Q_{i}^{(m+1)}$ and $Q_{i}^{(m+1)} \cap S_{m}^{\prime}=P_{i}^{(m)}$. Also, the ramification index of $Q_{i}^{(m)}$ over $Q_{i}^{(m+1)}$ is 1 and the ramification index of $Q_{i}^{(m+1)}$ over $P_{i}^{(m)}$ is $p$. Thus $\phi_{m}: C l\left(S_{m+1}\right) \rightarrow C l\left(S_{m}\right)$ sends $Q_{i}^{(m+1)}$ to $Q_{i}^{(m)}$ and $\phi_{m}^{\prime}: C l\left(S_{m}^{\prime}\right) \rightarrow$ $C l\left(S_{m+1}\right)$ sends $P_{i}^{(m)}$ to $p Q_{i}^{(m+1)}$.

By (1.2), (1.3) and (1.6) it follows that the $\operatorname{ker} \phi_{m}$ is isomorphic to a subgroup of $W_{m}$. By (5.5) and the proof of (4.6), we have the $\operatorname{ker} \phi_{m}$ has order at most $p^{q-1}$. 
Proceeding by induction we have that the primes $Q_{i}^{(m)}$ generate $C l\left(S_{m}\right)$ and are each of order $p^{m}$, hence the same is true of the primes $P_{i}^{(m)}$ in $S_{m}^{\prime}$. Since $\phi_{m}^{\prime}: C l\left(S_{m}^{\prime}\right) \rightarrow C l\left(S_{m+1}\right)$ is injective by (3.5), we see that the elements $p^{m} Q_{i}^{(m+1)}$ are a $Z / p Z$-basis for $\operatorname{ker} \phi_{m}$. Since the ramification index of $Q_{i}^{(m)}$ over $Q_{i}^{(m+1)}$ is $1, \phi_{m}$ is surjective, and the conclusion of the theorem follows.

Corollary 7. $C l\left(X_{m}\right)$ is generated by the codimension one cycles on $X_{m}$ defined by $u_{i}=0,1 \leqq i \leqq q-1$.

Proof. By (2.2) and (5.6).

8. Let $A$ be a Krull ring of characteristic $p \neq 0$ with quotient field $k$. Let $h_{1}, \cdots, h_{r+1} \in A\left[x_{1}, \cdots, x_{n}\right]$ satisfy all of the conditions of (2.1) and (2.5) as elements of $k\left[x_{1}, \cdots, x_{n}\right]$. Let $T=A\left[x_{1}, \cdots, x_{n+1}\right] /\left(h_{1}, \cdots, h_{r}, x_{n+1}^{p}-h_{r+1}\right)$. Assume $T$ is a Krull ring.

Corollary 9. Let $T$ be as in (5.8), $C l(T)$ is isomorphic to $C l(A) \oplus\left(\bigoplus_{i=1}^{q-1} Z / p Z\right)$.

Proof. Let $U$ be the multiplicative set of nonzero elements of $A$. Since $A$ is a Krull ring, so is $S=U^{-1} T$. By a theorem of Nagata ([7], theorem 6.3, page 21 ), we obtain an exact sequence

$$
0 \longrightarrow \mathrm{Cl}(A) \longrightarrow \mathrm{Cl}(\mathrm{T}) \longrightarrow \mathrm{Cl}(\mathrm{S}) \longrightarrow 0 .
$$

The surjection $C l(T) \rightarrow C l(S)$ is a split surjection. Thus $C l(T) \cong C l(A) \oplus C l(S)$.

Corollary 10. Let $R$ be as in (2.1) and assume $g \in k^{[n]}$ is homogeneous such that $\bar{g}$ is irreducible in $R$. Let $Y \subset A_{k}^{m}$ be defined by the equations $h_{1}=\cdots=h_{r}$ $=x_{n+1}^{p m}-g=0$, where $m$ is a positive integer. Then the coordinate ring of $Y$ is factorial if and only if $Y$ is regular in codimension one.

\section{References}

[1] Atiyah, M.F. and Macdonald, I.G., Introduction to Commutative Algebra, AddisonWesley, 1969.

[2] Fossum, Robert, The Divisor Class Group of a Krull Domain, Springer-Verlag, 1973.

[3] Hartshorne, Robin, Algebraic Geometry, Springer-Verlag, 1977.

[4] Jacobson, Nathan, Lectures in Abstract Algebras III, Springer-Verlag, 1964.

[5] Lang, Jeffrey, Purely inseparable extensions of unique factorization domains, Kyoto Journal, 26 (1990) 453-471.

[6] - The divisor classes of the hypersurface $z^{p m}=G\left(x_{1}, \cdots, x_{n}\right)$, Tran. Amer. Math. Soc., 278 (1983).

[7] Samuel, Pierre, Lectures on unique factorization domains, in Tata Lecture Notes, Tata Inst. of Fundamental Res. Bombay, 1964. 